\title{
CONF-970503--13
}

\section{A High-DC-Voltage GaAs Photoemission Gun: Transverse Emittance and Momentum Spread Measurements Dof ER/40/50--1/2/}

\author{
D. Engwall, C. Bohn, L. Cardman, B. Dunham, D. Kehne, R. Legg, H. Liu, \\ M. Shinn, and C. Sinclair \\ Thomas Jefferson National Accelerator Facility \\ 12000 Jefferson Avenue, Newport News, VA 23606
}

\section{Abstract}

We have built a high-DC-voltage photoemission gun and a diagnostic beamline permitting us to measure rms transverse emittance $\left(\tilde{\varepsilon}_{x}\right)$ and rms momentum spread $(\delta)$ of short-duration electron pulses produced by illuminating the cathode with light from a mode-locked, frequencydoubled Nd:YLF laser. The electron gun is a GaAs photocathode source designed to operate at $500 \mathrm{kV}$. We have measured $\tilde{\varepsilon}_{x}$ and $\delta$ for conditions ranging from emittancedominated to space-charge-dominated. We report these measurements as functions of microbunch charge for different beam radii, pulse lengths, and voltages/field gradients at the cathode, and compare them with PARMELA calculations.

\section{INTRODUCTION}

We have designed a $500 \mathrm{kV}$ GaAs photoemission gun with the intent of exploring the limits of high-DC-voltage photocathode sources. The aim is to demonstrate that such a gun can work reliably, to investigate experimentally the transverse and longitudinal properties of the beams produced, and to compare the measurements with PARMELA predictions to ascertain the validity of the code in the charge and energy regime explored. The highly uniform cathode field, the ability to decouple beam energy from accelerating gradient, and the ability to control both radial and temporal beam profiles (including beam length) through the drive laser makes this gun a platform uniquely suited to support our studies. Once characterized, the gun will serve as the electron source for the FEL currently under construction at Jefferson Lab [1].

We have built the gun, measured $\tilde{\varepsilon}_{x}$ and $\delta$ at 250 and $300 \mathrm{kV}$ (after processing to 300 and $335 \mathrm{kV}$, respectively), and analyzed the data to compare with those values predicted by PARMELA. The reduced voltage was necessary due to ceramic punch-throughs that occured at $400 \mathrm{kV}$. The punch-throughs, believed due to a combination of field emission (from the cathode support tube) and an improper choice of ceramic material, were easily sealed, but re-opened at voltages of $300-350 \mathrm{kV}$. New ceramics [2] and a new cathode support tube recently allowed the gun to process to $400 \mathrm{kV}$ and run at $350 \mathrm{kV}$. In the remainder of this paper we describe the experiment, simulations, and results of our measurements.

\section{THE EXPERIMENT}

\subsection{Experimental Apparatus}

The apparatus used in this experiment consists of the electron gun and diagnostic beamline. The layout of the gun and beamline is shown in figure 1.

The electron gun operates in a horizontal position. Its high voltage terminal is isolated by two ceramics stacked in series. The GaAs cathode wafer resides just inside the flattened end of a ball-shaped cathode which is supported from the high voltage terminal by a tube. The gun has planar anode-cathode geometry so that the field on the surface of the GaAs wafer at $500 \mathrm{kV}$ is $10 \mathrm{MV} / \mathrm{m}$, uniform to $1 \%$ over a $1 \mathrm{~cm}$ diameter. Optional inclusion of a spacer nipple at the base of the ceramic stack reduces the cathode field to $6.0 \mathrm{MV} / \mathrm{m}$ at $500 \mathrm{kV}$. A large-bore, water-cooled solenoid and RF-shielded gate valve immediately follow the gun vacuum chamber.

The beamline extends about $2 \mathrm{~m}$ beyond the valve. Immediately after the valve is the light box, a device that directs incident laser light onto the photocathode and outcouples light reflected from the wafer into a beam dump. The beam diagnostics reside in three vacuum crosses. A six-way cross houses a beam viewer and a scanning, $\sim 50 \mu \mathrm{m}$ wide vertical slit that renders transmitted beamlets emittance dominated. A $45^{\circ}$ dipole magnet follows the slit cross. After the magnet, the straight and branched sections of the beamline contain a five-way cross holding a beam viewer and wire scanner, and terminate in an isolated Faraday cup. The dipole horizontally images the slit onto wire scanner 2 , yielding a spectrometer with $22.5 \mathrm{~cm}$ of dispersion. Current readouts for the Faraday cups and wire scanners are provided by Keithley 485 picoammeters.

The beam is centered through the solenoid and beamline and the average charge per pulse is determined from the current intercepted in the straight-ahead Faraday cup. For the emittance measurement, we use a variant of the twoslit method $[3,4]$. We scan the slit horizontally (in the $x$ direction) across the beam, stopping at $\sim 13$ locations, and record the current profile of each transmitted beamlet with wire scanner 1. After background subtraction, these current profiles are used in conjunction with the slit locations to calculate $\tilde{\varepsilon}_{x}$ using $\tilde{\varepsilon}_{x} \equiv \sqrt{\left\langle x_{0}^{2}\right\rangle\left\langle x_{0}^{\prime 2}\right\rangle-\left\langle x_{0} x_{0}^{\prime}\right\rangle^{2}}$; where $x_{0}$ and $x_{0}^{\prime}$ are, respectively, the beam's horizontal coordinate and angle of divergence at the slit, and \langle\rangle signifies a current-weighted average. The rms normalized emittance is obtained in the ordinary manner: $\tilde{\varepsilon}_{x, n}=\beta \gamma \tilde{\varepsilon}_{x}$, with $\beta$ 


\section{DISCLAIMER}

This report was prepared as an account of work sponsored by an agency of the United States Government. Neither the United States Government nor any agency thereof, nor any of their employees, make any warranty, express or implied, or assumes any legal liability or responsibility for the accuracy, completeness, or usefulness of any information, apparatus, product, or process disclosed, or represents that its use would not infringe privately owned rights. Reference herein to any specific commercial product, process, or service by trade name, trademark, manufacturer, or otherwise does not necessarily constitute or imply its endorsement, recommendation, or favoring by the United States Government or any agency thereof. The views and opinions of authors expressed herein do not necessarily state or reflect those of the United States Government or any agency thereof. 


\section{DISCLAMMTR}

Portions of this document may be illegible in electronic image products. Images are produced from the best available original document. 


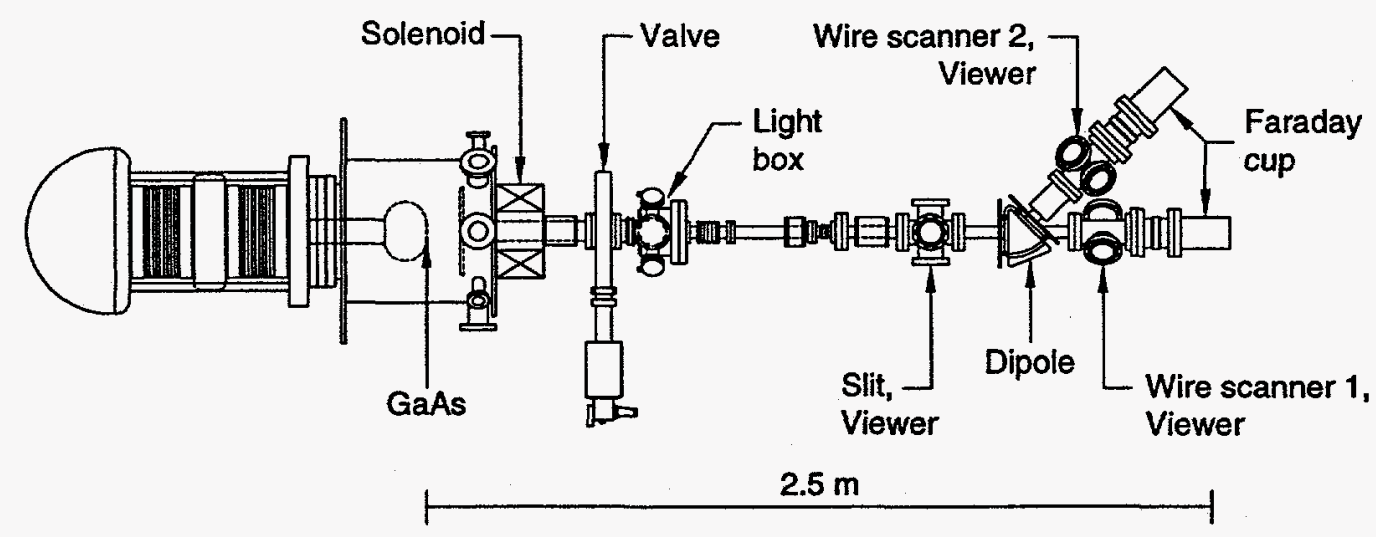

Figure 1: An overhead view of the gun (6 MV/m configuration) and the diagnostic beamline.

and $\gamma$ the usual relativistic factors. For the momentumspread measurement, we energize the dipole, place the slit in the center of the beam, and record the transmitted beamlet's current profile with wire scanner 2. After background subtraction, the profile is used to calculate $\delta$ using $\delta \equiv \sqrt{\left\langle x_{2}^{2}\right\rangle} / \mathcal{D}$; where $x_{2}$ is the beam's horizontal coordinate at wire scanner $2, \mathcal{D}$ is the spectrometer's dispersion, and we exploit the slit narrowness and the point-to-point focusing of the spectrometer.

\subsection{Experimental Conditions}

We measure $\tilde{\varepsilon}_{x}$ and $\delta$ as functions of microbunch charge ( $q$ ) for different combinations of beam radius, pulse length, and voltages (field gradients) at the cathode. We run the gun at 250 and $300 \mathrm{kV}$, with 3.0 and $3.6 \mathrm{MV} / \mathrm{m}$ cathode field strengths. The laser emits pulses with assumedGaussian temporal profiles of rms width $\sigma_{t}$. By changing the modelocker operating temperature or detuning the laser-cavity length, we obtain two stable pulse widths: $\sigma_{t} \approx 20$ and $25 \mathrm{ps}$. We change the electron beam radius $(R)$ by imaging three different diameter laser apertures onto the cathode, repeatably obtaining beam radii of 0.61 , 1.06 , and $1.95 \mathrm{~mm}$. Due to the limited amount of experimental time ( $<100$ hours) and the length of time ( $~ 1$ hour) required to complete a measurement at a single charge, we used PARMELA to generate the solenoidal field values $(B)$ for our experiment.

\section{THE SIMULATIONS}

We have simulated the experimental conditions as closely as possible using the standard Jefferson Lab version of PARMELA [6] with a slightly modified particle generation scheme. In our version, macroparticles are tracked using the McDonald point-to-point space charge algorithm [7] and the Liu variable-particle-size factor [6]. The modifications cause macroparticles to be emitted in an arbitrary, user-defined radial profile with a Maxwellian velocity distribution.

The electron bunches are simulated from the cathode to the slit using the same $B$ values as the experiment. Limiting the maximum particle size factor to $10[8]$ prevents over-shielding of the macroparticles. Use of POISSONgenerated electric and magnetic field profiles for the gun and solenoid assures the proper treatment of aberrations in these elements. The radial and longitudinal profiles of the macroparticle distribution are identical to those of the laser beam at the photcathode except that the longitudinal profile is truncated at $\pm 3 \sigma_{t}$. Finally, the macroparticles are emitted with a thermal energy of $\frac{1}{2} k_{B} T=0.18 \mathrm{eV}$. This value equates the simulated and measured values of $\tilde{\varepsilon}_{x}$ in the low-charge (emittance-dominated) regime for $R=1.95 \mathrm{~mm}$, and compares favorably to Dunham's value of $0.153 \pm 0.010 \mathrm{eV}$ [5].

\section{RESULTS}

In this section we present some of the results of our measurements. We estimate the statistical error in $\tilde{\varepsilon}_{x}(\delta)$ to be $\pm 5 \%( \pm 1.5 \%)$. We determine the statistical error by comparing repeated measurements at selected charges throughout the measurement range. We calculate an upper bound of $\pm 10 \%$ and $\pm 6 \%$ for the non-recoverable systematic error in the $\tilde{\varepsilon}_{x}$ and $\delta$ measurements, respectively. The bound includes contributions from baseline subtraction, finite sampling effects, determination of slit/wire positions and separations, and determination of the spectrometer dispersion. PARMELA simulations of the measurement method indicate an upper bound of $-15 \%$ for the recoverable systematic error in both measurements. Recoverable systematic errors arise from finite slit and wire widths, and "bent" wires. During vacuum bakeout, the wires in our scanners relaxed, resulting in a $\sim 6^{\circ}$ inclination of the wire with respect to the slit. These errors are deemed recoverable because future analysis will correct for them.

Figure 2 shows data taken for $R=1.06 \mathrm{~mm}$ : at $250 \mathrm{kV}$ with $\sigma_{t}=19 \mathrm{ps}$ and $\sigma_{t}=25 \mathrm{ps}$; and at $300 \mathrm{kV}$ with $\sigma_{t}=20 \mathrm{ps}$. Other data looks quite similar.

In terms of determining $\tilde{\varepsilon}_{x}$, PARMELA agrees quite well with experiment at the lowest charges, underestimates the value by $\sim 25 \%$ at higher charges, overestimates the value by $\sim 50 \%$ at even higher charges, and agrees quite well again with experiment at the highest charges. We believe the shape of the experimental curves, as well as 


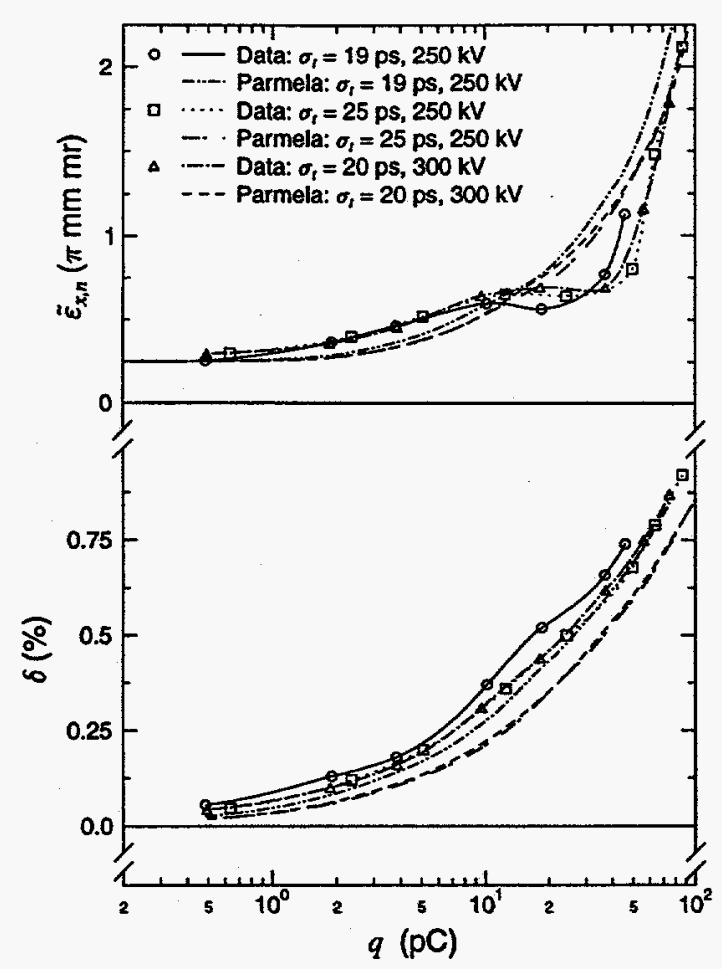

Figure 2: Results for measurements with $R=1.06 \mathrm{~mm}$ showing the effects of pulse length and accelerating potential (cathode field) variation.

the agreement between the data and PARMELA simulations, to be the result of differing levels of solenoidal compensation for different $q$. To support our contention, we introduce figure 3 , which shows $\tilde{\varepsilon}_{x}$ as a function of $B$ for $R=0.61 \mathrm{~mm}, \sigma_{t}=20 \mathrm{ps}, q=18.7 \mathrm{pC}$, and a beam energy of $250 \mathrm{kV}$.

From inspection of the experimental curve in figure 3 , we see that if $B$ is too large for the beam space charge, solenoidal compensation is negligible and the experimental values exceed the simulation values. As $B$ drops and becomes better matched to the beam space charge, increased solenoidal compensation causes the experimental value to dip below the simulation values and flatten. As $B$ decreases further, it is too small for the beam space charge; solenoidal compensation dissipates and the experimental value rises towards the simulation curve again.

The behavior just described qualitatively matches that of the experimental $\tilde{\varepsilon}_{x}$ curves in figure 2 , implying that the Parmela-generated solenoid values used in the experiment are too large for the beam space charge at low $q$, nearly matched to the beam space charge at intermediate $q$, and too small for the beam space charge at high $q$. The differing levels of solenoidal compensation induced by these effects cause the the experimental $\bar{\varepsilon}_{x}$ values to exceed the simulation values at low $q$ (once space charge effects become non-negligible), to level off and dip below the simulation values at intermediate $q$, and to rebound towards the simulation value at high $q$. Given the obvious insensitivity to solenoidal compensation that PARMELA shows in figure 3 , such errors in $B$ are not surprising.

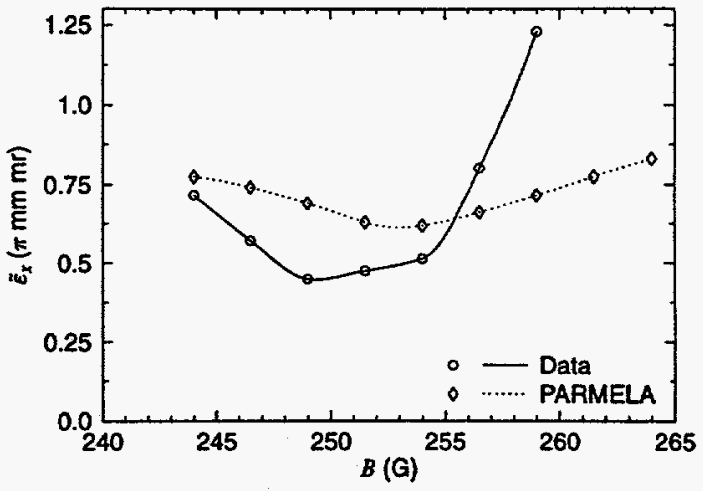

Figure 3: Results for measurements with $R=0.61 \mathrm{~mm}$, $\sigma_{t}=20 \mathrm{ps}$, and $q=18.7 \mathrm{pC}$, showing the dependence of $\tilde{\varepsilon}_{x}$ on $B$.

In terms of predicting $\delta$, PARMELA underestimates the value by $50-60 \%$ at lower charges, improving to underestimate the value by $\sim 10 \%$ at higher charges.

\section{CONCLUSIONS}

In this charge and energy regime, PARMELA is able to predict $\tilde{\varepsilon}_{x}$ and $\delta$ to within $50 \%$. The code displays a marked insensitivity to solenoidal compensation effects, possibly accounting for much of the discrepancy in predicting $\tilde{\varepsilon}_{x}$. Further research is necessary to determine the cause of this insensitivity.

\section{ACKNOWLEDGEMENTS}

Work supported by USDOE under contract DE-AC0584-ER 40150 and by USNSF under contract PHY-8921146. We thank Richard Hill, Timothy Siggins, and Dawn Venhaus for their help.

\section{REFERENCES}

[1] C.L. Bohn, "Recirculating Accelerator Driver for a HighPower Free-Electron Laser: A Design Overview", these Proceedings.

[2] F. Liu, et al., "A Method of Producing Surface Conduction on Ceramic Accelerator Components Using Metal Ion Implantation", these Proceedings.

[3] C. LeJeune and J. Aubert, in Applied Charged Particle Optics, A. Septier, Ed., Advances in Electronics and Electron Physics, Supp. 13A, 159-259 (1980).

[4] B. Dunham, et al., "An Emittance Measurement System for a Wide Range of Bunch Charges", these Proceedings.

[5] B.M. Dunham, Ph.D. thesis, University of Illinois at UrbanaChampaign (1993).

[6] H. Liu, et al., Nucl. Instr. and Meth., A358 475 (1995).

[7] K.T. McDonald, IEEE Trans. on Electron Devices, 352052 (1988).

[8] H. Liu, CEBAF Technical Note 94-040 (1994). 